

\section{Sumário}

Editorial: ConstituCiOnalismo latino-AMERICANO: O QUE TEMOS EM COMUM? .15

Editorial: Constitucionalismo Latinoamericano: ¿Qué TENEMOS EN COMÚN? 17 Luís Roberto Barroso e Patrícia Perrone Campos Mello

Seção I: Poder Constituinte

As Constituições latino-AMERICANAS ENTRE A VIDA E A MORTE: POSSIBILIDADES E LIMITES DO PODER DE EMENDA

Luís Roberto Barroso e Aline Osorio

Criação constitucional sem Poder Constituinte: Os limites conceituais do poder de SUBSTITUIÇÃO OU REVISÃO DA CONSTITUIÇÃO

Carlos Bernal Pulido

Quem Conta Como naÇão? A ExClusão de temáticas LGBTI nas asSEMbleias CONSTituintes de Brasil e Colômbia

Rafael Carrano Lelis, Marcos Felipe Lopes de Almeida e Waleska Marcy Rosa

Em Defesa da PaRTicipaÇão: ANálise da iniciativa POPUlar Para alteraÇão da Constituição no Brasil E No EquAdor

Ilana Aló Cardoso Ribeiro e Lílian Márcia Balmant Emerique

Reflexões críticas sobre o Processo Constituinte Equatoriano de Montecristi (2007-2008)

E. Emiliano Maldonado

SeÇão II: Jurisdição Constitucional e Democracia

LA REVISIÓN JUDICIAL EN DEMOCRACIAS DEFECTUOSAS 154 Roberto Gargarella

Constituição E PluRalismo JURÍdico: A POSIÇÃo PARTICUlar do Brasil NO CONTEXTO LATINO-AMERICANO

Ana Paula Gonçalves Pereira de Barcellos

As CONSTITUiÇões LATINO-AMERICANAS PELAS LENTES DAS CORTES CONSTITUCiONAIS: A FORÇA NORMATIVA E O ROMANTISMO DOS PREÂMBULOS 185 Arnaldo Sampaio de Moraes Godoy e Carlos Frederico Santos 
REDES SOCIALES, JUSTICIA CONSTITUCIONAL Y DELIBERACIÓN PÚBLICA DE CALIDAD: LECCIONES DEL Plebiscito por la PAZ EN Colombia...........................................................203 Jorge Ernesto Roa Roa

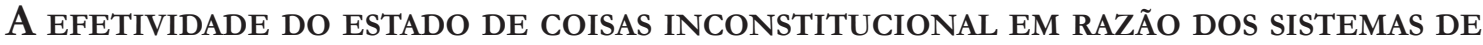
monitoramento: uma aNÁlise comparativa ENTRE Colômbia E Brasil 218

Aléssia Barroso Lima Brito Campos Chevitarese, Ana Borges Coêlho Santos e Felipe Meneses Graça

Seção III: Constitucionalismo Transformador e "Ius Constitucionale Commune" na AmÉRica LATina 231

O MANDATO TRANSFORMADOR DO Sistema INTERAMERICANO: LEgALIDADE E LEGITIMIDADE DE UM PROCESSO JURISGENÉTICO EXTRAORDINÁRIO 233 Armin von Bogdandy

CONSTITUCIONALISMO, TRANSFORMAÇÃO E RESILIÊNCIA DEMOCRÁTICA NO BRASIL: O IUS Constitucionale Commune na América Latina tem uma Contribuição a oferecer? .. 254 Patrícia Perrone Campos Mello

UM PROJETO COMUM PARA A AMÉRICA LATINA E OS IMPACTOS DAS EMPRESAS EM DIREITOS HUMANOS .287

Danielle Anne Pamplona

O papel da Corte Interamericana de Direitos humanos na construção dialogada do Ius Constitutionale Commune na América Latina..............................................303 Ana Carolina Lopes Olsen e Katya Kozicki

Seção IV: Novo Constitucionalismo Latino-Americano

O papel da Corte Interamericana de Direitos humanos na construÇão dialogada do Ius Constitutionale Commune na América Latina.................................................334 Roberto Viciano Pastor e Rubén Martínez Dalmau

¡Que viva El Estado Plurinacional!: ¿Y lo socioambiental? 351 Anibal Alejandro Rojas Hernández, aula Harumi Kanno, Heline Sivini Ferreira e Adriele Fernanda Andrade Précoma

O NOVO CONSTITUCIONALISMO LATINO-AMERICANO: ANÁLISE MARXISTA DA INVISIBILIZAÇÃO DA LUTA DE CLASSES NAS INVESTIGAÇÕES JURÍDICAS CRÍTICAS .365

Daniel Araújo Valença, Ronaldo Moreira Maia Júnior e Rayane Cristina de Andrade Gomes

O NOVO CONSTITUCIONALISMO LATINO-AMERICANO: ANÁLISE MARXISTA DA INVISIBILIZAÇÃO DA LUTA DE CLASSES NAS INVESTIGAÇÕES JURÍDICAS CRÍTICAS .382

Adriele Andrade Précoma, Heline Sivini Ferreira e Rogério Silva Portanova 
O direito À ÁGUA NAs Constituições da América do Sul: Elementos comuns e traços DISTINTIVOS.

Thiago Rafael Burckhart e Milena Petters Melo

Direitos Humanos na América Latina: avanços e desafios inerentes À atual CONJUNTURA POLÍTICA

Paulo Renato Vitória e Gabriela Maia Rebouças

O Novo Constitucionalismo na América latina e Caribe, e a construção do direito À SAÚDE

Alethele de Oliveira Santos, Maria Célia Delduque e Moacyr Rey Filho

A qualidade da educaÇão para a EFETIVaÇão E Consolidação do novo CONSTITUCIONALISMO LATINO-AMERICANO NO EQUADOR E NA BOLÍVIA.

Manuel Rodrigues de Sousa Junior e Luigi Bonizzato

A diversidade Cultural segundo o entendimento da Corte InTERAmericana de Direitos Humanos e o Novo Constitucionalismo Latino-Americano transformador. 476 Bianor Saraiva Nogueira Júnior, Deicy Yurley Parra Flórez e Ulisses Arjan Cruz dos Santos

UN APORTE A LA PROTECCIÓN DE LOS DERECHOS HUMANOS EN LATINOAMÉRICA: LA ACTIVIDAD del Ombudsman Criollo en tribunales de justicia 493 Juan Pablo Díaz Fuenzalida

SEÇÃo VI: Povos Indígenas

El DERECHO FUNDAMENTAL A LA IDENTIDAD CULTURAL DE LOS PUEBLOS INDÍGENAS: UN DERECHO-MATRIZ Y FILTRO HERMENÉUTICO PARA LAS CONSTITUCIONES DE AMÉRICA LATINA: LA JUSTIFICACIÓN 514 Juan Jorge Faundes

Povos INDígenas E A (AUSÊNCIA DE) JUSTIÇA DE TRANSIÇÃo BRASILEIRA: UMA ANÁLISE À LUZ DO CONSTITUCIONALISMO PLURALISTA LATINO-AMERICANO Jamilly Izabela de Brito Silva e Sílvia Maria da Silveira Loureiro

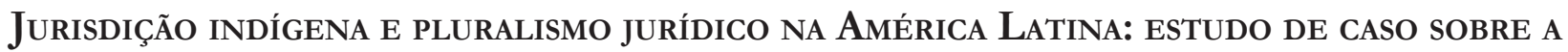
JUSTIÇA WAIWAI .558 João Vitor Cardoso e Luiz Guilherme Arcaro Conci

O NOVO CONSTITUCIONALISMO LATINO-AMERICANO E OS POVOS INDÍGENAS: A VisÃo Do DiREITO A PARTIR dOS CALEIDOSCÓPIOS E DOS MONÓCUlOS 577 Lucas Silva de Souza, Valéria Ribas do Nascimento e Isadora Forgiarini Balem 


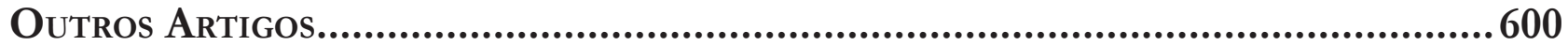

BUILDING TRUST IN COLLABORATIVE PROCESS OF VILLAGE FUND POLICY IMPLEMENTATION (A Case Study at Luwuk District of Banggai Regency) .........................................602 Rahmawati halim 


\title{
Redes sociales, justicia constitucional y deliberación pública de calidad: lecciones del plebiscito por la paz en Colombia*
}

\author{
Redes sociais, justiça constitucional e \\ deliberação pública de qualidade: lições do \\ plebiscito pela paz na Colômbia
}

Jorge Ernesto Roa Roa**

\section{Resumen}

Desde una perspectiva deliberativa, este artículo analiza el plebiscito realizado en Colombia en el año 2016 para ratificar el Acuerdo de Paz. En el texto se argumenta que el diseño de esa consulta tuvo un alto costo deliberativo que repercutió en la forma como se dejó abierto un profundo desacuerdo social. Además, se infieren algunas lecciones sobre la influencia negativa de las redes sociales, las fake news y la posverdad en mecanismos de participación popular con diseños deficientes. Asimismo, se delimita el rol del poder judicial frente a consultas con problemas deliberativos y se argumenta a favor de la adopción de medidas estratégicas de largo plazo que permitan la estabilidad institucional en momentos de crisis o emergencia. Finalmente, en el texto se propone una arquitectura constitucional compleja que permita asegurar la igualdad en los procesos de participación ciudadana y obtener una respuesta confiable frente a desacuerdos sociales complejos y profundos.

Palabras-clave: Deliberación, acuerdo de paz, Colombia, redes sociales, posverdad.

\section{Resumo}

O presente artigo analisa o plebiscito realizado na Colômbia, no ano de 2016, que tinha por objeto a ratificação do Acordo de Paz, com base em uma perspectiva deliberativa. O texto argumenta que o desenho dessa consulta teve um alto custo deliberativo, que repercutiu na forma por meio da qual se manteve aberto um profundo desacordo social. Além disso, se extraem algumas lições sobre a influência negativa das redes sociais, as fake news e a pós-verdade com relação a mecanismos de participação popular com desenhos deficientes. Ainda assim, se identifica o papel do Poder Judiciário no que respeita a consultas com problemas deliberativos e se argumenta no sentido da adoção de medidas estratégicas de longo prazo que permitam a estabilidade institucional em momentos de crise ou emergência. Finalmente, se propõe, no texto, uma arquitetura constitucional complexa que permita assegurar a igualdade nos processos de participação cidadã e obter uma re-

** Doctor en Derecho (summa cum laude) por la Universidad Pompeu Fabra de Barcelona. Profesor de derechos humanos y derecho constitucional comparado de la Universidad Externado de Colombia. Contacto: jorge.roa@ uexternado.edu.co. 
sposta confiável diante de desacordos sociais complexos e profundos.

Palavras-chave: Deliberação, acordo de paz, Colômbia, redes sociais, pós-verdade.

\section{Introducción}

La tesis que se defiende en este artículo es que existe una conexión entre el diseño institucional de los procesos electorales o de los mecanismos de participación ciudadana con la potencial influencia de las redes sociales e internet en esas decisiones. Esa tesis descriptiva implica una tesis normativa, de acuerdo con la cual, es necesario diseñar el proceso electoral y de participación ciudadana de manera que esos procesos logren la mayor deliberación con el fin de combatir las influencias negativas de las redes sociales y potenciar las influencias positivas de esos mecanismos.

El artículo se divide en cuatro partes. En la primera parte se describe la tensión entre el consentimiento electoral informado -que propende por el mayor flujo posible de la información- y el consentimiento electoral cualificado -que propende por una información de calidad-. En la segunda parte se estudia el caso del plebiscito convocado en Colombia para refrendar el acuerdo de paz firmado con la guerrilla de las FARC en 2016. El objetivo de ese análisis es verificar la manera como la tensión descrita en la sección anterior se presentó en ese plebiscito, la influencia de las redes sociales en ese proceso de participación ciudadana y las lecciones que ofrece ese caso. En especial, se mantendrá que los errores del diseño institucional de ese mecanismo lo hicieron deficitario en términos deliberativos y, por ende, altamente permeable a la influencia negativa de las redes sociales.

En tercer lugar, se analiza el rol del poder judicial frente a procesos electorales o de participación ciudadana con fuerte influencia de las redes sociales, el populismo, la posverdad y las fake news. Finalmente, se esboza una propuesta de diseño institucional de los mecanismos de participación ciudadana con el objetivo de que estos reflejen un modelo deliberativo, potencien las ventajas de las redes sociales, minimicen sus riesgos y permitan conocer la voluntad real de la sociedad frente a un desacuerdo social relevante ${ }^{1}$.

\section{La tensión entre el libre mercado de las ideas (consentimiento electoral informado) y la información de calidad (consentimiento electoral cualificado) en el plebiscito por la paz en Colombia}

En materia de libertad de expresión y acceso a la información en los procesos electorales se presenta una tensión que está directamente relacionada con el poder informativo de las redes sociales. Por una parte, existe la pretensión de que en esos periodos circule la mayor cantidad de información posible. Eso significa que se haga efectivo el libre mercado de las ideas, se pronuncien y escuchen todas las voces sobre temas de interés público y exista el mayor escrutinio a los candidatos y propuestas ${ }^{2}$. El objetivo es que el tiempo electoral sea un tiempo esencialmente deliberativo y que esa deliberación sea robusta, profunda y rica. Por esa razón, incluso se tolera la crítica, la ofensa y la expresión incómoda ${ }^{3}$.

Las redes sociales resultan idóneas para lograr esa primera finalidad porque permiten una comunicación

WALDRON, Jeremy. Derecho y desacuerdos. Marcial Pons: Madrid, 2009.

2 NACIONES UNIDAS. Informe del Relator Especial sobre la promoción y protección del derecho a la libertad de opinión y de expresión, Frank La Rue. A/HRC/26/3. 2014.

ORGANIZACIÓN DE LOS ESTADOS AMERICANOS. Comisión Interamericana de Derechos Humanos. Declaración conjunta sobre medios de comunicación y elecciones. R 29/09. 2009. Disponible en: http://www.oas.org/es/cidh/expresion/showarticle. asp?artID=745\&lID=2. Acceso en: 8 mayo 2019 . 
fluida, ágil, rápida y gratuita o, al menos, de bajo costo para el usuario. Además, la multiplicidad de fuentes contrarresta los efectos perversos de los monopolios informativos y la influencia de los intereses económicos en los medios $\square$ tradicionales- de comunicación ${ }^{4}$. Se podría concluir que en los periodos electorales son bienvenidas las redes sociales para que exista la mayor cantidad de fuentes de información y el consentimiento popular sea plenamente informado 5 .

Por otra parte, no es suficiente con que el consentimiento electoral sea informado, sino que este debe ser cualificado. Eso implica contar con información de calidad en la que exista el mayor nivel de veracidad y se combatan, entre otras, las fake newss. Además, la información debe ser accesible para todo el público, es decir, se debe presentar de manera sencilla, objetiva y debe ser el producto de investigaciones de calidad ${ }^{6}$.

En relación con esta segunda pretensión, las redes sociales no son siempre idóneas porque presentan altos riesgos que cuestionan la posibilidad de cumplir estos objetivos. Por ejemplo, las cuentas falsas, los trolls, la manipulación de los algoritmos, las tendencias emotivas, la movilización de escritorio (clicktivismo) que desestimula la movilización social y el fomento de la abstención electoral ${ }^{7}$.

Esa tensión entre el consentimiento electoral informado y el cualificado se presentó en el plebiscito convocado por el Gobierno de Colombia con el fin de ratificar el acuerdo de paz negociado con la guerrilla de las FARC en la Habana (Cuba) y firmado en Cartagena en el año 2016.

Por una parte, existía un afán desmedido del Gobierno del presidente Juan Manuel Santos por ratificar el acuerdo de paz. Aunque la ratificación popular del acuerdo de paz no era obligatoria desde el punto de vista jurídico, esta adquirió esa condición en virtud de tres factores. En primer lugar, el presidente se comprometió públicamente a consultar al pueblo cuando finalizaran las negociaciones con la guerrilla ${ }^{8}$. En segundo lugar, la guerrilla exigió un mecanismo de refrendación popular del acuerdo final mediante una asamblea constituyente ${ }^{9}$. A pesar de que, durante las negociaciones, el grupo guerrillero renunció a la idea de la asamblea constituyente, este mantuvo su exigencia de que el acuerdo fuera refrendado popularmente por algún mecanismo de los existentes en la Constitución de 1991: consulta popular, plebiscito o referendo. En tercer lugar, la aprobación popular mayoritaria del acuerdo de paz en la votación del plebiscito fue establecida como el requisito necesario para activar el procedimiento legislativo especial o fast track que permitiría aprobar las leyes y reformas constitucionales necesarias para implementar el acuerdo final de paz ${ }^{10}$.

El Gobierno diseñó un mecanismo de refrendación popular del acuerdo de paz ad hoc basado en el plebiscito establecido en la Constitución de 1991. Mediante la Ley Estatutaria 1806 de 2016, el Congreso de la República autorizó al presidente a convocar un plebiscito con las siguientes características: i) umbral de aprobación del 13\%, ii) carácter vinculante a efectos del desarrollo legal del acuerdo, iii) tiempo de campaña de tres meses porque el plebiscito no podía ocurrir antes de un mes, ni después de cuatro meses contados

\footnotetext{
WILLIAMS, Christine; GULATI, Girish. Social networks in political campaigns: Facebook and the congressional elections of 2006 and 2008. New Media and Society, v. 15, n. 1, p. 52-71, 2013.

5 BAKER, Andy; AMES, Barry; RENNO, Lucio. Social context and campaign volatility in new democracies: networks and neighborhoods in Brazil's 2002 Elections. American Journal of Political Sciencie, v. 50, n. 2, p. 382-399, 2006.

6 HALU, Arda; ZHAO, Kun; BARONCHELLI, Andrea; BIANCONI, Ginestra. Connect and win: the role of social networks in political elections. EPL Europhysics Letters, v. 102, n. 1, p. 1-5, 2013.

TUMASJAN, Andranik; SPRENGER, Timm; SANDNER, Philipp; WELPE, Isabell. Predicting elections with twitter: what 140 characters reveal about political sentiment. AAAI Publications, Fourth International AAAI Conference on Weblogs and Social Media, p. 178-185, 2010. Disponible en: https://www.aaai.org/ocs/index.php/ICWSM/ICWSM10/paper/view/1441/1852. Acceso en: 8 mayo 2019 .

8 SANTOS, Juan Manuel. La batalla por la paæ: el largo camino para poner fin al conflicto con la guerrilla más antigua del mundo. Madrid: Planeta, 2019.p. 529, 531.

9 DE LA CALLE, Humberto. Revelaciones al final de una guerra: testimonio del jefe negociador del gobierno colombiano en La Habana. Bogotá: Debate, 2019.

10 ROA ROA, Jorge Ernesto. Control constitucional del 'fast track y deliberación pública de calidad. Ámbito Jurídico, 2017. Disponible en: https://www.ambitojuridico.com/noticias/constitucional-y-derechos-humanos/control-constitucional-del-fast-tracky-deliberacion. Acceso en: 8 mayo 2019.
} 
desde el momento en el que el presidente enviaba la convocatoria al Congreso de la República. La configuración de ese plebiscito fue avalada por la Corte Constitucional en las sentencias C-379 de 2016 y C-699 de $2016^{11}$.

La configuración especial del plebiscito tenía altos costos en términos deliberativos. Sin embargo, la prisa desmedida del Gobierno -como suele ocurrir con las prisas institucionales- era razonable por varios factores. En primer lugar, la mayor parte de la guerrilla se había desmovilizado y estaba concentrada en algunas veredas a la espera de comenzar el proceso de sometimiento a la justicia e incorporación a la vida civil. Además, el Gobierno del presidente Santos había superado la mitad de su segundo periodo (2014-2018), lo cual reducía su poder e influencia sobre el Congreso de la República. Asimismo, la proximidad de las elecciones parlamentarias y presidenciales amenazaban las mayorías afines al proceso de paz dentro del parlamento. Finalmente, el Estado debía empezar a ocupar los territorios dejados por la guerrilla. Todo esto sugería un proceso de refrendación expedito, en el que el poder de las redes sociales era esencial para difundir el acuerdo, fomentar el debate, lograr la movilización y convocar al pueblo a las urnas ${ }^{12}$.

Ahora bien, el costo de oportunidad deliberativo de ese mecanismo expedito de refrendación popular del acuerdo de paz aumentaba en virtud de varios factores. Por una parte, es importante recordar que el acuerdo final tenía una extensión superior a las 300 páginas, se refería a todas las materias de la vida nacional, desde la agricultura, la economía o el acceso a la justicia hasta aspectos culturales o deportivos ${ }^{13}$. Se trataba, sin duda, de un acuerdo que cerraba un conflicto armado de más de cincuenta años, cuya implementación requería una serie de reformas legales y constitucionales de amplia trascendencia. Todo esto sugería un proceso de deliberación pausada y de refrendación compleja y extendida en el que las redes sociales representaban más riesgos que beneficios.

En el caso del plebiscito por la paz se asumió el costo deliberativo y prevaleció la urgencia de refrendar el acuerdo de paz para iniciar su implementación. Con el mecanismo diseñado por el Gobierno, aprobado por el Congreso y avalado por la Corte Constitucional, se puso en marcha una campaña breve e intensa que terminó con el apretado resultado contrario a la refrendación del acuerdo de paz. Los resultados definitivos del plebiscito realizado el 2 de octubre de 2016 fueron los siguientes: a favor del SI 49,78\% (6.377.482 votos) y a favor del NO 50,21\% (6.431.376 votos). El índice de participación fue de 37,43\%, lo cual implica que participaron 13.066.047 de las 34.899.945 personas habilitadas para votar en Colombia en ese momento ${ }^{14}$. A continuación, se indican algunas lecciones de esta tensión y de esta historia.

\section{Lecciones de una consulta popular precipitada en la que hubo mucho espacio para la posverdad, las fake news y la mentira en las redes sociales}

El diseño, la campaña y los resultados del plebiscito por la paz de 2016 en Colombia permiten inferir algunas conclusiones (lecciones) sobre la conexión entre el diseño institucional de este tipo de procesos de

\footnotetext{
1 ROA ROA, Jorge Ernesto. La acción pública de constitucionalidad a debate. Bogotá: Universidad Externado de Colombia, 2015 . p. 3-6. 12 ROA ROA, Jorge Ernesto. Deliberación y dilación en el control de constitucionalidad. Ámbito Jurídico, 2017. Disponible en: https://www.ambitojuridico.com/noticias/analisis/constitucional-y-derechos-humanos/deliberacion-y-dilacion-en-el-control-de. Acceso en: 8 mayo 2019.

13 El texto del Acuerdo final para la terminación del conflicto y la construcción de una paz estable y duradera suscrito entre el Gobierno de Colombia y las Fuerzas Armadas Revolucionarias de Colombia FARC-EP se puede consultar en: http://www. altocomisionadoparalapaz.gov.co/procesos-y-conversaciones/Documentos\%20compartidos/24-11-2016NuevoAcuerdoFinal.pdf. Acceso en: 7 mayo 2019.

14 Los resultados oficiales de las votaciones en el plebiscito se pueden consultar en línea en la página web de la Registraduría Nacional del Estado Civil. Allí se ofrecen datos desagregados por departamentos: https://elecciones.registraduria.gov.co/pre_ plebis_2016/99PL/DPLZZZZZZZZZZZZZZZZZ_L1.htm. Acceso en: 26 abr. 2019.
} 
consulta popular y la influencia de las redes sociales. A continuación, se mencionan cuatro de esas lecciones que deberían generar un proceso de reflexión sobre la manera de construir y diseñar los mecanismos de participación popular basados en una ingeniería constitucional moderna, compleja y enfocada en la manera de resolver desacuerdos sociales complejos sin despreciar la deliberación.

\subsection{En realidad, nadie ganó deliberativamente el plebiscito por la paz}

La primera lección que se infiere de todo el proceso de refrendación del acuerdo de paz realizado en 2016 es que ninguna de las opciones puede reivindicar una victoria clara. Dicho de otra manera, no es plausible determinar la manera como fue resuelto el desacuerdo social sobre la ratificación (o no) del acuerdo de paz.

Por supuesto que, desde el punto de vista estrictamente matemático y en virtud de la alta fiabilidad del sistema electoral colombiano de ese momento, la opción favorable a la refrendación del acuerdo de paz (el SI) perdió. Sin embargo, el triunfo matemático de la opción contraria a la refrendación de los acuerdos de paz (el NO) quedó deslegitimado, no tanto por su escasa mayoría sobre el SI -53.894 votos- como por la estrategia de campaña utilizada por sus promotores.

Es precisamente en este punto en el que la calidad de la deliberación pública que precede al acto de depositar un voto en una urna adquiere su mayor relevancia. Por eso, el hecho de que la estrategia de los promotores de la opción contraria a la refrendación de los acuerdos de paz se hubiese basado en la difusión de mentiras deslegitima su ya menguada victoria electoral. En efecto, como fue reconocido por el gerente de la campaña a favor de la opción NO, se utilizaron mentiras tanto sobre el contenido de las negociaciones (i.e. el gobierno cedió todo a la guerrilla) y el texto o contenido del acuerdo (i.e. se incluyó la ideología de género; se estableció un modelo político y económico propio del castrochavismo; el acuerdo le entrega el país a las FARC; el acuerdo le asigna dinero, le concede pensiones y les dona casas a los guerrilleros y el acuerdo es un pacto de impunidad) como sobre el futuro del país (i.e. Colombia se va a convertir en Venezuela cuando se implemente el acuerdo de paz).

De conformidad con su estrategia, los promotores de la opción contraria a la refrendación del acuerdo de paz generaron contenidos unificados en torno a mensajes emotivos que canalizaban toda la rabia, apatía e indignación popular que la población colombiana ha sentido tradicionalmente contra la guerrilla de las FARC. Esos sentimientos se redirigieron en contra del acuerdo de paz que, paradójicamente, ponía fin a la estela de violencia dejada por ese grupo armado. Así lo reconoció el jefe de la campaña por el NO, Juan Carlos Vélez, en una entrevista para un medio de comunicación que fuera concedida apenas cuatro días después de celebrado el plebiscito. A la pregunta: "La campaña del SI fue basada en la esperanza de un nuevo país: ¿Cuál fue el mensaje de ustedes?” El señor Vélez contestó lo siguiente: "La indignación. Estábamos buscando que la gente saliera a votar verraca" ${ }^{\prime 15}$.

Eso significa, entre otras cosas, que la estrategia consistía precisamente en apagar o desviar la deliberación sobre el acuerdo de paz. En concreto, de lo que se trataba era de evitar discutir el contenido del acuerdo mientras que se argumentaba en torno a temas que no tenían relación directa con el objeto de la consulta. Por ejemplo, se difundió la idea de que votar en contra de la refrendación del acuerdo de paz equivalía a: oponerse a aspectos tan disímiles como la interrupción voluntaria del embarazo, rechazar la denominada ideología de género o evitar que se redujeran las pensiones a los jubilados.

Por esa razón, si lo que interesa es algo mucho más importante que contar papeletas en una urna porque se encuentra más valioso analizar el grado de información, debate, discusión, acuerdos y respuestas sociales frente a un desacuerdo social, resulta difícil reconocer la victoria de alguna de las dos partes en el plebiscito

\footnotetext{
15 VÉLEZ, Juan Carlos. Estábamos buscando que la gente saliera a votar verraca. El colombiano. Medellín, 2016. Disponible en: https://www.elcolombiano.com/colombia/acuerdos-de-gobierno-y-farc/entrevista-a-juan-carlos-velez-sobre-la-estrategia-de-lacampana-del-no-en-el-plebiscito-CE5116400. Acceso en: 8 mayo 2019.
} 
por la paz.

\subsection{Los límites de las redes sociales frente al consentimiento electoral informado y cualificado}

La anterior lección permite inferir una segunda relacionada con los límites de las redes sociales para contribuir a la formación de un consentimiento electoral informado y cualificado. Esto ocurre especialmente porque la veracidad no ocupa un lugar central en algunos medios digitales, la gratuidad de estos permite la difusión masiva de información distorsionada y existen fuertes límites de esos entornos para explicar y deliberar sobre desacuerdos sociales complejos.

En primer lugar, la veracidad es un concepto que ocupa un segundo lugar en las redes sociales. Por el contrario, la capacidad para despertar emociones suele ser la prioridad en la red porque esta, a su vez, tiene mayor capacidad de movilización. A la falta de información veraz se agrega que una parte de esa información no es contrastada y proviene de fuentes anónimas. En el caso del plebiscito por la paz en Colombia, el propio líder de la campaña por el NO confesó que, en este aspecto, su estrategia se basó en mentiras y en imágenes falsas que, además, incitaban el odio de los ciudadanos ${ }^{16}$.

En materia de veracidad existen dos asimetrías que afectan a las redes sociales. Por una parte, la primera asimetría consiste en que, una vez difundido un mensaje con un contenido falso, la carga de desmentir tal mensaje y eliminarlo del debate público es muy alta para quien resulta afectado frente a la rapidez y facilidad con la que este fue difundido. Como señala el propio expresidente de Colombia, Juan Manuel Santos:

Es muy difícil defenderse de una mentira, y de medias verdades que, por incompletas, son las mejores aliadas de la falsedad. Mucho más en estos tiempos de internet y redes sociales, cuando vuelan a la velocidad del viento. Y debo admitir que no dimensionamos, ni el Gobierno ni los amigos del proceso de paz, cuánto podían pesar estas mentiras en los ciudadanos, y cómo podían influir en su decisión de apoyar o no el acuerdo en el plebiscito. No acabábamos de refutar una, cuando ya estaban circulando diez más. Y así fueron ganando un terreno que no logramos cubrir con la pedagogía ${ }^{17}$.

La segunda asimetría se produce entre la apelación a las emociones y la veracidad. Esta consiste en que, una vez que se ha movilizado emocionalmente, no existe información veraz que pueda cambiar la posición de quien ha sido motivado por sus sentimientos. En el plebiscito sobre el acuerdo de paz en Colombia, tanto los amigos del proceso con las FARC como la oposición apelaron al miedo de volver a la guerra (los primeros) o de que el país fuera gobernado por la guerrilla (los segundos) para movilizar a su electorado. Al menos los primeros han reconocido su error al haber acudido, dentro de su estrategia de campaña, al miedo y no a la esperanza y a la información básica sobre el acuerdo de paz ${ }^{18}$.

La segunda arista de esta lección es que la gratuidad de las redes sociales no siempre es una oportunidad para el acceso a información de calidad. En este ámbito, la gran promesa de las redes sociales era que la gratuidad iba a democratizar la información frente al gran poder económico de unos grupos con intereses políticos. En concreto, las redes permitirían disminuir el poder informativo de las élites económicas o políticas. El ciudadano tomaría su ordenador o su teléfono para informarse de acuerdo con sus preferencias, empoderarse y movilizarse. Sin embargo, en contra de lo esperado, la gratuidad ha representado una oportunidad para quienes pueden contratar empresas que administran cuentas falsas y trolls, para el influencer que difunde dolosamente información falsa y para la consolidación de grupos económicos e informativos que

\footnotetext{
16 VÉLEZ, Juan Carlos. Estábamos buscando que la gente saliera a votar verraca. El colombiano. Medellín, 2016. Disponible en: https://www.elcolombiano.com/colombia/acuerdos-de-gobierno-y-farc/entrevista-a-juan-carlos-velez-sobre-la-estrategia-de-lacampana-del-no-en-el-plebiscito-CE5116400. Acceso en: 8 mayo 2019.

17 SANTOS, Juan Manuel. La batalla por la paæ: el largo camino para poner fin al conflicto con la guerrilla más antigua del mundo. Madrid: Planeta, 2019. p. 532.

18 SANTOS, Juan Manuel. La batalla por la pa\%: el largo camino para poner fin al conflicto con la guerrilla más antigua del mundo. Madrid: Planeta, 2019. p. 534-535.
} 
se han adaptado a las nuevas tecnologías.

Eso significa que el acceso gratuito o a bajo costo a las redes sociales no conlleva efectos transformadores, ni facilita el acceso a la información política por parte de personas vulnerables ${ }^{19}$. De nuevo, el propio director de la campaña en contra de la refrendación del acuerdo de paz confiesa como la gratuidad de las redes sociales habilitó la manipulación de la información durante la campaña. A la pregunta: $\square$ ¿Con cuánto dinero se hizo la campaña?”, contestó:

[] Fue una campaña hecha con las uñas. En el partido del Centro Democrático y en la corporación que creamos "La paz es de todos" logramos recaudar $\$ 1.300$ millones, principalmente de 30 empresas y 30 personas naturales. Fue muy difícil conseguir respaldo y los bancos no estaban preparados. Sin embargo, el No fue la campaña más barata y efectiva en mucho tiempo. Su costo-beneficio es muy alto ${ }^{20}$.

La misma decepción ocurre cuando se analizan las asimetrías entre el centro y la periferia y la influencia diferenciada de las redes sociales en las regiones más apartadas del país. Por último, pero no menos importante, es imprescindible reconocer que las redes sociales tienen límites para explicar y deliberar sobre desacuerdos sociales complejos. El intercambio ágil en las redes sociales puede conducir al siguiente dilema: reducir la complejidad para ampliar la cobertura de un mensaje o mantener una deliberación compleja centrada en unos cuantos participantes. En el caso del plebiscito por la paz en Colombia, quienes intentaron enfrentar la campaña de mentiras que se realizaba en las redes sociales contra el acuerdo de paz mediante argumentos sólidos pero complejos, finalmente fracasaron porque a los promotores de la opción NO les "resultó más fácil traducir al lenguaje de las redes sociales la simplicidad de los argumentos opositores que las complejas razones del oficialismo"21.

\subsection{Es imprescindible fortalecer la protesta social, la movilización social y reivindicar al periodismo como actividad profesional}

La tercera lección sobre la influencia de las redes sociales en el plebiscito por la paz en Colombia es que es imprescindible recuperar la protesta social, la movilización ciudadana y el periodismo independiente y de calidad en momentos de profundos desacuerdos sociales. Desde luego, ninguno de esos factores puede eliminar por completo el riesgo de manipulación, pero sí tiene la capacidad de reducir la desinformación y desarticular movimientos sistemáticos que apagan la deliberación.

En este ámbito, la gran promesa de las redes sociales era que estas iban a crear, a su vez, redes mundiales que conectaran a los movimientos sociales. No hay duda de que existen ejemplos de que esto ha ocurrido. Sin embargo, el verdadero sentido de esa expectativa era que el ciudadano tomaría su ordenador o su teléfono para manifestar su indignación y convocar a otros ciudadanos en su misma condición. El resultado de ese proceso serían las primaveras árabes, los movimientos de indignados y campañas como me too u Occupy Wall Street.

Esta promesa se invirtió con la preocupación por el desenfreno, la intensidad, la pugnacidad y la virulencia de las discusiones en las redes sociales frente a una apatía y abstención ante la movilización social y la participación electoral. El activismo de escritorio (clicktivismo) es el factor con el que el ciudadano suele compensar su falta de movilización real. Al final, las experiencias de movilización social coordinadas a través de las redes sociales son tan maravillosas como verdaderamente excepcionales e insostenibles.

\footnotetext{
19 BERNAL TRIVIÑ O, Ana Isabel. Tecnología, redes sociales, política y periodismo. ¿Pluralidad informativa o efecto bumerán? Cuadernos.info, n. 36, p. 191-205 p. 192, 2015.

20 VÉLEZ, Juan Carlos. Estábamos buscando que la gente saliera a votar verraca. El colombiano. Medellín, 2016. Disponible en: https://www.elcolombiano.com/colombia/acuerdos-de-gobierno-y-farc/entrevista-a-juan-carlos-velez-sobre-la-estrategia-de-lacampana-del-no-en-el-plebiscito-CE5116400. Acceso en: 8 mayo 2019.

21 GONZÁLEZ, María Fernanda. La «posverdad» en el plebiscito por la paz en Colombia. Revista Nueva Sociedad, n. 269, p. 114126, 2016.
} 
En este panorama, habría que reivindicar al periodismo como una actividad profesional con unos códigos éticos y profesionales frente a los desafíos de las redes sociales. Un error común en el que se incurre como producto de la emoción que aparejan los avances digitales es pensar que la deliberación de calidad se puede dejar librada a lo que ocurra en las redes. Se trata, desde luego, de una creencia que perjudica a la propia deliberación democrática. En este aspecto, resultan equivalentes tanto los errores de omitir lo que se discute en las redes -que es una parte de nuestra deliberación colectiva- como suponer que esta solo se desarrolla en tales entornos digitales.

El modelo deliberativo -que se revela contra la idea de que existe un enemigo interno o externo, contra la mentira dolosa, contra los ataques al Estado de Derecho y a los Derechos Humanos o contra la idea de que todas la élites e instituciones públicas son corruptas- rescata las virtudes de una prensa libre, vigorosa e independiente frente al modelo populista que la desacredita, utiliza las redes sociales para anular el debate, crea enemigos y oculta deliberadamente información relevante.

\subsection{Lección final: en materia de diseño institucional, nunca fue más cierto que del afán solo queda el cansancio}

De acuerdo con el diseño constitucional del plebiscito por la paz en Colombia, el triunfo del NO carecía de efectos vinculantes. Sin embargo, según se indicó previamente, la activación del mecanismo de refrendación (fast track) del acuerdo de paz se vinculó al triunfo del SI en la consulta ciudadana. Con el resultado favorable a la opción NO, salieron a relucir todos los problemas del diseño inicial del plebiscito. Esto ocasionó que se profundizara la tensión entre la necesidad de profundo debate público, la prisa institucional y la presión por la falta de tiempo para implementar los acuerdos antes de que finalizara el Gobierno del presidente Santos.

Por esa razón, después de conocidos los resultados del plebiscito, hubo que abrir una fase de doble negociación. Por una parte, se debió negociar con los representantes del NO los puntos que debían ser modificados del acuerdo. Por otra parte, se debió renegociar con la guerrilla de las FARC esas mismas modificaciones. Todo lo anterior se realizó con una prisa inédita que negó toda posibilidad a un debate abierto y profundo sobre los errores cometidos al diseñar el primer mecanismo de refrendación, el significado de los resultados del plebiscito y la forma de enmendar todo el proceso. Por el contrario, se optó por la vía rápida de realizar unas cuantas reuniones con voceros del NO, trasladar sus objeciones a la guerrilla y pactar un nuevo acuerdo. Este, a su vez, fue firmado en el teatro Colón de Bogotá, cuestionadamente refrendado por el Congreso y su implementación comenzó un año después de lo planeado ${ }^{22}$.

Como era previsible, los líderes de la campaña por el NO siempre han reclamado el déficit de refrendación del segundo acuerdo en virtud de que este no fue sometido nuevamente a la consideración del pueblo, sino que se ratificó mediante lo que se denominó refrendación popular indirecta ${ }^{23}$. La refrendación parlamentaria, que fue avalada por la Corte Constitucional con base, inter alia, en las sentencias C-699 de 2016, C-160 de 2017 y C-007 de 2018, ha sufrido duros reveses en virtud del triunfo electoral del partido de oposición al acuerdo de paz en las elecciones presidenciales que dieron la victoria a Iván Duque y las elecciones parlamentarias en las que aumentó el número de congresistas contrarios al acuerdo de paz. A partir de ese punto, se ha abierto un proceso de revisión legislativa de las normas de implementación, con lo cual, la Corte Constitucional ha debido asumir una labor de defensa de los avances normativos que aplicaron el acuerdo de paz. Se trata, como se dirá en la siguiente sección, del rol propio de un tribunal constitucional en un contexto de constitucionalismo transicional.

22 ROA ROA, Jorge Ernesto. La sustitución constitucional, el 'fast track' y el Acuerdo Final de Paz. Ámbito Jurídico, 2017. Disponible en: https://www.ambitojuridico.com/noticias/analisis/constitucional-y-derechos-humanos/la-sustitucion-constitucionalel-fast-track-y. Acceso en: 8 mayo 2019.

23 Ver, entre otras: Ley 1820 de 2016 (artículo 1) y Proposición 83, Gaceta del Congreso, año XXVI. 


\section{El rol del juez en transiciones y posverdad}

Uno de los grandes debates del constitucionalismo colombiano contemporáneo gira en torno a la potencial existencia de un constitucionalismo transicional, los rasgos esenciales de ese (¿nuevo?) constitucionalismo y sus consecuencias concretas sobre la organización del poder público y los derechos de los ciudadanos. Una de las ideas centrales que respalda la existencia de un constitucionalismo transicional es el diseño de instituciones y procedimientos para permitir la refrendación, implementación y desarrollo de todas las transformaciones necesarias para consolidar la transición del conflicto armado hacia la desmovilización armada de los antiguos grupos guerrilleros. En este aspecto, tanto el constitucionalismo transformador como el constitucionalismo transicional ostentan estrechos puntos de conexión.

En el marco del constitucionalismo transicional, una de las principales cuestiones por resolver es el papel de los tribunales dentro del proceso de transición. Afortunadamente, sobre este aspecto existen múltiples casos en el constitucionalismo comparado que permiten analizar el papel que juegan los tribunales durante estos complejos periodos sociales. Con base en la experiencia de Sudáfrica, por ejemplo, Rosalind Dixon y Theunis Roux han señalado que los tribunales constitucionales pueden jugar un rol de impulso (transformador) o un rol de conservación (conservador) en los procesos de transición. Desde luego, también existe la posibilidad de que los jueces se ubiquen en una posición intermedia y gradúen la velocidad de la transición para conseguir el equilibrio entre los viejos y nuevos proyectos políticos y sociales ${ }^{24}$.

Una de las principales lecciones de los estudios comparados es que, si los tribunales constitucionales apuestan por un papel transformador y de impulso, es importante que cuenten con aliados institucionales y sociales para avanzar esas transformaciones. Los aliados pueden ser los demás poderes del Estado (Gobierno y Congreso), las organizaciones sociales y la ciudadanía en general. Además, es deseable que la Corte Constitucional desarrolle construcciones jurisprudenciales sólidas que permitan el avance colectivo de la transición. Por ejemplo, es importante contar con una serie de precedentes sobre la deferencia razonable que la Corte debe tener con el legislador. Este es un buen mecanismo para realizar el control de constitucionalidad efectivo sobre las normas de transición y, al mismo tiempo, avanzar en alianza con las demás instituciones del Estado ${ }^{25}$.

Desde luego, el hecho de que la Corte Constitucional asuma la función de impulsar la transición no resuelve todos los problemas, no soluciona todos los dilemas, ni materializa todas las transformaciones que requiere la transición material. Sin embargo, es importante que la Corte comprenda que, si no impulsa la transición de manera conjunta con el Congreso -vale decir, no es deferente con las reformas aprobadas por el Congreso- la carga posterior de proteger la transición y lograr otros avances será responsabilidad casi exclusiva del propio tribunal.

El anterior riesgo es todavía más acuciante cuando cambian las condiciones políticas y las instituciones favorables a la transición pasan a ser ocupadas por movimientos adversos a ese proceso de cambio. Por esa razón, es deseable potenciar el momento cooperativo y dialógico para avanzar en la transición con el fin de crear los precedentes adecuados para impulsar las transformaciones, reaccionar frente a los problemas emergentes y enfrentar los retrocesos promovidos por Gobiernos menos comprometidos con la transición.

Entre otras cosas, eso implica un cambio en la forma de construir la jurisprudencia constitucional, de manera que, al mismo tiempo, se piense a largo plazo y se mantenga la sensibilidad por el periodo político actual. También es un momento adecuado para reconocer que algunas herramientas deben mutar o evo-

24 DIXON, Rosalind; ROUX, Theunis. Marking constitutional transitions: the law and politics of constitutional implementation in South Africa. University of New South Wales Law Research Series, n. 64, p. 1-18, 2018.

25 ROA ROA, Jorge Ernesto. Los retos constitucionales del proceso de paz en Colombia: la fase instrumental de ratificación o refrendación y la implementación de los acuerdos de paz. Documentos de Trabajo del Departamento de Derecho Constitucional de la Universidad Externado de Colombia, n. 47, p. 1-25, 2015. 
lucionar. Por ejemplo, la Corte Constitucional debe aceptar que el juicio de sustitución no está acabado, ni tampoco el fundamento de su implementación; mucho menos los casos en los que procede o el tipo de enmienda que pretende evitar.

Asimismo, el reto de una judicatura que apueste por impulsar la transición es hacer un esfuerzo por mantenerse como un actor jurídico, superar las tradiciones jurídicas formalistas consolidadas antes de la transición, evitar las interpretaciones originalistas de la Constitución y habilitar la evolución del sistema constitucional. El objetivo final es la construcción de fundamentos jurídicos sólidos para las decisiones e intervenciones que debe realizar el tribunal del constitucionalismo transicional.

Ahora bien, la pregunta concreta es cómo pueden actuar los jueces para impulsar la transición y asegurar, simultáneamente, que hay un amplio debate y deliberación en todas las etapas de ese proceso. En el caso del plebiscito por la paz de 2016 en Colombia, por ejemplo, cuál ha debido ser el rol de los jueces para proteger el consentimiento informado y cualificado de la ciudadanía frente a la estrategia de desinformación e instrumentalización de las redes sociales utilizada por los adversarios del acuerdo de paz.

Desde luego, existen casos claros en los que el juez puede i) desbloquear la deliberación cuando hay censura oficial por medio de la no concesión de licencias, la publicidad pública, la censura y el bloqueo de portales de internet o redes sociales; ii) proteger la honra, el buen nombre y la intimidad en casos extremos de fake news o discurso del odio; y iii) limitar el ejercicio de la libertad de expresión cuando este implica una incitación a la violencia contra un candidato, partido político o idea.

Sin embargo, en casos más sutiles de instrumentalización estratégica de las redes sociales -como el del plebiscito por la paz en Colombia o el Brexit en el Reino Unido- los tribunales tienen poco margen de maniobra. Quizá lo máximo que puede hacer el juez es levantar la mano y decir: jalguien ha mentido!, pero no tiene el poder de anular los resultados de consultas, como el plebiscito por la paz o el Brexit, con base en ese argumento. Se trata de una respuesta débil e insatisfactoria, pero con profundos efectos simbólicos y respetuosa, en últimas, del principio democrático.

En este último sentido fue precisamente la actuación del Consejo de Estado de Colombia al admitir la demanda interpuesta por dos ciudadanos que solicitaron la nulidad de los actos administrativos que consolidaron los resultados del plebiscito por la paz. El argumento de los demandantes era que se había ejercido violencia psicológica contra los electores. La prueba esencial de esa violencia psicológica era la entrevista (aquí citada en varias oportunidades) del líder de la campaña del NO en la que reconocía haber utilizado las redes sociales para mentir o distraer la discusión pública. El fundamento jurídico de esa demanda fue el artículo 275-1 de la Ley 1437 de 2011 o Código de Procedimiento Administrativo y de lo Contencioso Administrativo. Allí se establecía que una causal de nulidad electoral era que se hubiera ejercido cualquier tipo de violencia en contra del elector.

El Consejo de Estado declaró probada la causal de nulidad invocada por los demandantes y decretó algunas medidas cautelares. La principal medida cautelar consistió en ordenar al Congreso que avanzara en la implementación del acuerdo de paz mediante el fast track y exhortó a la Corte Constitucional a priorizar las demandas de constitucionalidad sobre la vigencia del procedimiento legislativo especial. En la fundamentación de su decisión, el Consejo de Estado de Colombia hizo lo que se acaba de indicar, esto es, levantar la mano y decir: jalguien ha mentido! En efecto, así se lee en la providencia:

[] Es así que cuando mediante maniobras engañosas, se obtiene que la ciudadanía vote un plebiscito en determinado sentido "o incluso que se abstenga de hacerlo, si ello es el resultado de un comportamiento que coarta la libertad electoral” lo que se logra al final no es más que un resultado espurio, en la medida en que está edificado sobre la destrucción de los pilares sobre los cuales se levanta la democracia, y que son los mismos cuya preservación se extrema a través de los principios pro hominum (humanidad), pro electoratem (electorado) y pro sufragium (electores).

[] todo engaño que anule la libertad del electorado para escoger autónomamente entre las opciones 
sometidas al mecanismo de participación en cuestión, entre los que destacan circunstancias como i) la pululación de mentiras expuestas de formas masiva y sistemática, ii) la creación de un clima rodeado de información sesgada y deliberadamente manipulada, iii) así como la distorsión o tergiversación de la verdad mediante el uso de propaganda, o cualquier otro medio, o iv) la omisión deliberada en la revelación de aspectos necesarios para la cabal comprensión de los diferentes puntos del "acuerdo de paz", y v) su manipulación en todos los niveles, provengan de quien provengan, son actos que constituyen violencia psicológica, cuya incidencia se expande isotrópicamente hacia todo el electorado ${ }^{26}$.

Esta decisión del Consejo de Estado y las sentencias de la Corte Constitucional que avalaron tanto el mecanismo de refrendación popular directa como los actos de refrendación indirecta del acuerdo de paz permitieron desatar el bloqueo institucional que había creado el inesperado resultado del plebiscito. En perspectiva, la judicatura asumió un rol de impulso de la transición en un contexto favorable en el que el Gobierno y el Congreso eran aliados del proceso de cambio. Ahora bien, de cara al futuro, no solo es importante evitar la judicialización completa de la paz ${ }^{27}$ sino que es muy relevante pensar los diseños institucionales y la arquitectura constitucional para asegurar la deliberación en los procesos que definen las diferencias más profundas dentro de la sociedad.

\section{Un diseño institucional que minimice los riesgos negativos de influencia de las redes sociales y potencie la deliberación}

El alcance de este artículo no permite medir la influencia exacta de las redes sociales en los resultados finales del plebiscito. No solo no existe un estudio de data y bigdata que haya hecho tal medición en Colombia, sino que hay un amplio desacuerdo sobre la forma de hallar la relación entre la difusión de mensajes (verdaderos o falsos) en las redes sociales y la expresión de la voluntad ciudadana en las urnas. Ahora bien, lo que sí se puede afirmar es que el resultado final del plebiscito de 2016 fue sorpresivo tanto para los promotores de ambas opciones como para todos los encuestadores y para el propio Gobierno. Aun así, no ha habido una sola voz seria que cuestione la fiabilidad del mecanismo electoral, por lo cual, una de las opciones es pensar, como se ha indicado en este texto, que la sorpresiva victoria de quienes se oponían a la refrendación del acuerdo de paz se debiera, en buena medida, a que su campaña tuvo un desempeño cualitativamente superior en las redes sociales ${ }^{28}$.

Si eso fue así, entonces desde el punto de vista de la ingeniería constitucional corresponde formular alguna respuesta de diseño institucional que minimice los riesgos de las redes sociales, potencie sus ventajas y, especialmente, amplíe la deliberación en momentos de amplio desacuerdo social. Eso significa que la tesis que aquí se defiende parte de la confianza en el diseño de los mecanismos electorales y de participación ciudadana como medio para limitar las influencias negativas de las redes sociales y potenciar sus ventajas en momentos de decisiones públicas.

Una modificación inmediata y mínima que podría cualificar el diseño de consultas populares -como el plebiscito por la paz en Colombia o el Brexit en el Reino Unido- consistiría en introducir la doble vuelta o doble refrendación. El proceso iniciaría con una primera campaña extendida, pausada y robusta en la que se debatirían todas las opciones sobre un determinado desacuerdo social. A esa campaña seguiría una primera votación de la que derivaría un primer resultado electoral. A partir de ese momento, habría un tiempo de reflexión de uno, tres, cinco o diez años, según corresponda con la dimensión del desacuerdo social. Entre

26 Consejo de Estado, Sección Quinta, Auto de medidas cautelares en el Radicado 11001-03-28-000-2016-00081-00, 19 diciembre 2016. p. 49 y 51.

27 GARGARELLA, Roberto. Some reservations concerning the judicialization of peace. Harvard International Law Journal Comment, v. 59, p. 1-19, 2019.

28 GONZÁLEZ, María Fernanda. La «posverdad» en el plebiscito por la paz en Colombia. Revista Nueva Sociedad, n. 269, p. 114126 , p. 114, 2016 
otras cosas, durante ese periodo se analizaría todo lo ocurrido hasta el resultado electoral inicial: las mayorías obtenidas, el nivel de abstención, la reacción nacional e internacional, el comportamiento de los mercados y, especialmente, las causas del veredicto electoral.

Posteriormente, se abriría un segundo periodo de deliberación y movilización de cara a una segunda votación. No resulta insólito esperar que la segunda etapa sea cualitativamente superior en términos deliberativos porque en esta se han analizado las posibles influencias perversas de las redes sociales en el resultado electoral inicial, la abstención se puede reducir en la segunda elección si se logra movilizar a las personas que no se movilizaron en la primera votación y se mantiene el interés público por el tema. Por el contrario, si el desacuerdo social deviene en irrelevante puede ocurrir que, en realidad, el asunto sometido a consulta fuera también irrelevante socialmente, producto de una coyuntura o que el desacuerdo no fuera tan profundo como para ameritar una consulta popular. Por esa razón, el segundo momento deliberativo debe ser lo más prolongado posible e, idealmente, más distendido que la campaña inicial.

La segunda votación puede dar lugar a dos escenarios. Por una parte, si el resultado 1 se confirma en el resultado 2, eso le confiere una mayor legitimidad al primero por la sostenibilidad de la mayoría a favor de una determinada respuesta social. Aunque la mayoría sea ajustada en ambos resultados, eso significa que el objeto de la consulta es un desacuerdo profundo de la sociedad y que la mejor manera de resolverlo era mediante la (doble) consulta popular.

Sin embargo, el resultado 2 puede ser contrario al resultado 1. En ese caso, surge un problema porque no hay un criterio para determinar cuál de los dos resultados debe prevalecer. Se podría pensar en la prevalecía del segundo resultado en virtud de que la etapa posterior a la primera elección agrega virtudes deliberativas a lo ocurrido en la primera votación. Sin embargo, lo que verdaderamente demuestra la contradicción entre el primer y el segundo resultado es que el objeto consultado refleja un desacuerdo genuino dentro de la sociedad sobre el que la propia población no tiene un criterio definitivo. Es decir, se prueba que no era bueno quedarse con el primer veredicto porque se trataba de una imagen parcial, precaria y fragmentada de la opinión pública sobre un determinado debate público.

Esta propuesta deja el desasosiego de no saber qué hacer en este segundo caso. Sin embargo, la respuesta es que es necesario dar tiempo para que la sociedad depure ese desacuerdo. Ninguna contradicción social es tan urgente que no pueda dar tiempo de espera a la madurez del debate y de la deliberación. Ni siquiera bajo los estados de emergencia o excepción es posible tomar decisiones inmediatas sin seguir unos determinados protocolos. Esos tiempos de espera no son formalidades o protocolos vacuos, sino que son condiciones fundamentales para mejorar las ventajas epistémicas de nuestras decisiones como sociedad.

Así lo demostró Stephen Holmes en 2009 al señalar que las situaciones en las que aparentemente se requiere una respuesta inmediata, rápida, eficaz, apresurada, efectiva y poco deliberada son, en realidad, momentos que exigen mayor pausa, seguir protocolos, acciones coordinadas, deliberación y contención. Holmes empezaba su artículo relatando la experiencia de su hija Alexa, quien sufrió un grave accidente que la dejó en estado de coma. Cuando la niña se encontraba en la sala de emergencias de la clínica, las enfermeras y profesionales que la atendieron actuaron bajo estrictos protocolos que dilataban la acción urgente e inmediata que la situación de la Alexa ameritaba. Por ejemplo, antes de transfundirle sangre, las enfermeras verificaban dos veces el nombre de la paciente y el tipo de sangre. Aunque todos los profesionales sabían que la vida de la paciente dependía de que ellos actuaran rápidamente, nunca dejaron de seguir los protocolos. Lo que esto enseña, dice Holmes, es que esos protocolos evitan que se comentan "errores fatales pero evitables bajo las presiones psicológicamente inquietantes del momento" ${ }^{\text {. }}$.

La idea es evitar lo que el propio Holmes denominó reacciones viscerales que son un mal sustituto de las reacciones estratégicas porque aquellas se basan en un supuesto falso que indica que cada emergencia

29 HOLMES, Stephen. In case of emergency: misunderstanding tradeoffs in the war on terror. California Law Review, v. 97, n. 2, p. 301-355. p. 301-302, 2009. 
requiere una reacción instantánea en lugar de una decisión de fondo basada en la consideración de todos los intereses involucrados y en un proceso sosegado de deliberación. De lo que se trata es de tomar la mejor decisión (pausada, deliberativa) que resuelva un profundo desacuerdo social. En momentos en que existe inestabilidad institucional es mejor adoptar decisiones que den pausa, estabilidad, control y no decisiones que profundicen la excepcionalidad, la angustia social y la desestabilización de los poderes públicos.

En este punto vale hacer la siguiente pregunta: ¿todo debe ser lentitud, pausa y deliberación en el diseño institucional? Desde luego que la respuesta es negativa porque también se deben implementar mecanismos de reacción digital rápida que permitan la comunicación directa entre los ciudadanos y los centros de decisión. En este ámbito son destacables los procesos constituyentes que se han realizado de manera digitalmente abierta y que han permitido a cualquier persona proponer, comentar o discutir el contenido de una política. Por ejemplo, en el caso de Islandia, los ciudadanos podían comunicar sus opiniones por correo electrónico o por Twitter, Facebook, YouTube y Flickr. En este ámbito, las instituciones públicas tienen el gran reto de implementar procedimientos de reacción por medio de las propias redes sociales para que el diálogo ocurra en el mismo nivel y con la fluidez que exigen los entornos digitales.

Finalmente, nada de lo dicho aquí debe llevar a pensar, como dijera el expresidente Juan Manuel Santos, que "en los referendos o plebiscitos la gente vota por todo menos por lo que se les está preguntando" 30 . Desde luego que existen muchos factores que determinan el acto de marcar una u otra opción en una papeleta. Sin embargo, el modelo deliberativo también se revela contra la idea de que el ciudadano es manipulable, ignorante o exclusivamente interesado.

Por el contrario, aquí se han resumido unas lecciones de un proceso esencialmente mal diseñado y se han sugerido algunas propuestas para evitar cometer el mismo error en el futuro. En cuanto eso sucede, el constitucionalismo transicional colombiano necesita enfrentar el hecho duro de que perdura un profundo desacuerdo social que no ha sido resuelto y que tiene que ver con la refrendación social del acuerdo de paz, el futuro de los arreglos hechos con la guerrilla y la mejor forma de avanzar la transición. Parece bastante como para considerarlo resuelto con una proposición en el Congreso, algunas normas aprobadas rápidamente y unas cuantas sentencias de la Corte Constitucional. El reto es, entonces, enorme y requiere de la mayor deliberación pública de calidad.

\section{Bibliografía}

BAKER, Andy; AMES, Barry; RENNO, Lucio. Social context and campaign volatility in new democracies: networks and neighborhoods in Brazil's 2002 Elections. American Journal of Political Sciencie, v. 50, n. 2, p. 382-399, 2006.

BERNAL TRIVIÑO, Ana Isabel. Tecnología, redes sociales, política y periodismo. ¿Pluralidad informativa o efecto bumerán? Cuadernos.info, n. 36, p. 191-205, 2015.

ORGANIZACIÓN DE LOS ESTADOS AMERICANOS. Comisión Interamericana de Derechos Humanos. Declaración conjunta sobre medios de comunicación y elecciones. R 29/09. 2009. Disponible en: http://www.oas. org/es/cidh/expresion/showarticle.asp?artID=745\&lID=2. Acceso en: 8 mayo 2019.

DE LA CALLE, Humberto. Revelaciones al final de una guerra: testimonio del jefe negociador del gobierno colombiano en La Habana. Bogotá: Debate, 2019.

DIXON, Rosalind; ROUX, Theunis. Marking constitutional transitions: the law and politics of constitutional implementation in South Africa. University of New South Wales Law Research Series, n. 64, p. 1-18, 2018.

30 SANTOS, Juan Manuel. La batalla por la paz: el largo camino para poner fin al conflicto con la guerrilla más antigua del mundo. Madrid: Planeta, 2019. p. 530. 
GARGARELLA, Roberto. Some reservations concerning the judicialization of peace. Harvard International Law Journal Comment, v. 59, p. 1-19, 2019.

GONZÁLEZ, María Fernanda. La «posverdad» en el plebiscito por la paz en Colombia. Revista Nueva Sociedad, n. 269, p. 114-126, 2016.

HALU, Arda; ZHAO, Kun; BARONCHELLI, Andrea; BIANCONI, Ginestra. Connect and win: the role of social networks in political elections. EPL Europhysics Letters, v. 102, n. 1, p. 1-5, 2013.

HOLMES, Stephen. In case of emergency: misunderstanding tradeoffs in the war on terror. California Law Review, v. 97, n. 2, p. 301-355, 2009.

NACIONES UNIDAS. Informe del Relator Especial sobre la promoción y protección del derecho a la libertad de opinión y de expresión, Frank La Rue. A/HRC/26/3. 2014.

ROA ROA, Jorge Ernesto. Control constitucional del 'fast track y deliberación pública de calidad. Ámbito Jurídico, 2017. Disponible en: https://www.ambitojuridico.com/noticias/constitucional-y-derechos-humanos/control-constitucional-del-fast-track-y-deliberacion. Acceso en: 8 mayo 2019.

ROA ROA, Jorge Ernesto. Deliberación y dilación en el control de constitucionalidad. Ámbito Jurídico, 2017. Disponible en: https://www.ambitojuridico.com/noticias/analisis/constitucional-y-derechos-humanos/ deliberacion-y-dilacion-en-el-control-de. Acceso en: 8 mayo 2019.

ROA ROA, Jorge Ernesto. La acción pública de constitucionalidad a debate. Bogotá: Universidad Externado de Colombia, 2015.

ROA ROA, Jorge Ernesto. La Corte Constitucional y el constitucionalismo transicional. Ámbito Jurídico, 2017. Disponible en: <https://www.ambitojuridico.com/noticias/columnista-impreso/constitucional-yderechos-humanos/la-corte-constitucional-y-el>. Acceso en: 8 mayo 2019.

ROA ROA, Jorge Ernesto. La sustitución constitucional, el 'fast track' y el Acuerdo Final de Paz. Ámbito Juridico, 2017. Disponible en: https://www.ambitojuridico.com/noticias/analisis/constitucional-y-derechoshumanos/la-sustitucion-constitucional-el-fast-track-y. Acceso en: 8 mayo 2019.

ROA ROA, Jorge Ernesto. Los retos constitucionales del proceso de paz en Colombia: la fase instrumental de ratificación o refrendación y la implementación de los acuerdos de paz. Documentos de Trabajo del Departamento de Derecho Constitucional de la Universidad Externado de Colombia, n. 47, p. 1-25, 2015.

SANTOS, Juan Manuel. La batalla por la paz: el largo camino para poner fin al conflicto con la guerrilla más antigua del mundo. Madrid: Planeta, 2019.

TUMASJAN, Andranik; SPRENGER, Timm; SANDNER, Philipp; WELPE, Isabell. Predicting elections with twitter: what 140 characters reveal about political sentiment. AAAI Publications, Fourth International AAAI Conference on Weblogs and Social Media, p. 178-185, 2010. Disponible en: https://www.aaai.org/ocs/ index.php/ICWSM/ICWSM10/paper/view/1441/185. Acceso en: 8 mayo 2019.

VÉLEZ, Juan Carlos. Estábamos buscando que la gente saliera a votar verraca. El colombiano. Medellín, 2016. Disponible en: https://www.elcolombiano.com/colombia/acuerdos-de-gobierno-y-farc/entrevistaa-juan-carlos-velez-sobre-la-estrategia-de-la-campana-del-no-en-el-plebiscito-CE5116400. Acceso en: 8 mayo 2019.

WALDRON, Jeremy. Derecho y desacuerdos. Marcial Pons: Madrid, 2009.

WILLIAMS, Christine; GULATI, Girish. Social networks in political campaigns: Facebook and the congressional elections of 2006 and 2008. New Media and Society, v. 15, n. 1, p. 52-71, 2013. 
Para publicar na revista Brasileira de Políticas Públicas, acesse o endereço eletrônico www.rbpp.uniceub.br

Observe as normas de publicação, para facilitar e agilizar o trabalho de edição. 